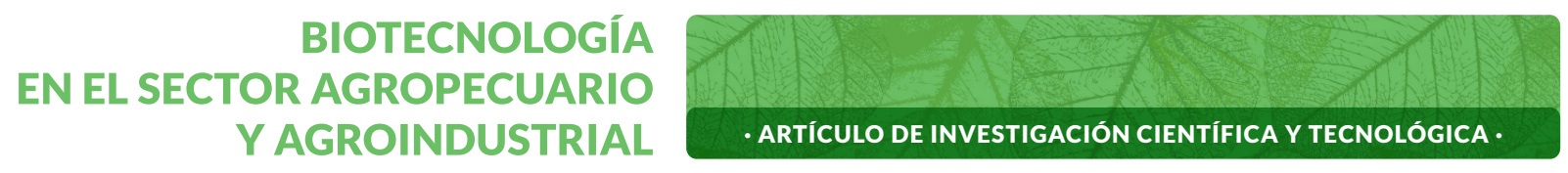

\title{
Estudio del biodiesel obtenido a partir de aceite de Sacha inchi (Plukenetia volubilis Linneo)*
}

\section{Study of biodiesel from Sacha inchi oil (Plukenetia volubilis Linneo)}

\author{
BASTO-FLOREZ, LUIS-EDUARDO'1; MILLÁN-ALVARADO, SEBASTIAN-AUGUSTO2; \\ MEDINA-CABALLERO, LUISA-FERNANDA ${ }^{3}$; MORA-VERGARA, LIA-ZAMARA4; \\ CABALLERO-HERNÁNDEZ, YULEISI-TATIANA ${ }^{5}$
}

Historial del artículo

Recibido para evaluación: 16 de Julio 2020

Aprobado para publicación: 4 de Noviembre 2021

* Título del Proyecto de origen: "Estudio del biodiesel obtenido a partir de aceite de sacha inchi (Plukenetia volubilis linneo)". Financiación: Instituto Universitario de la Paz. Culminación: abril 2019.

1 Instituto Universitario de la Paz (UNIPAZ), Escuela de Ingeniería Agroindustrial, Grupo de Investigación en Innovación, Desarrollo Tecnológico y Competitividad en Sistemas de Producción Agroindustrial (GIADAI). Esp. Tecnológico en control de calidad de biocombustibles líquidos. Barrancabermeja, Colombia. https://orcid.org/0000-0001-9550-3812

2 Instituto Universitario de la Paz (UNIPAZ), Escuela de Ingeniería Agroindustrial, Grupo de Investigación en Innovación, Desarrollo Tecnológico y Competitividad en Sistemas de Producción Agroindustrial (GIADAI). Esp. Tecnológico en control de calidad de biocombustibles líquidos. Barrancabermeja, Colombia. https://orcid.org/0000-0003-2600-8724

3 Instituto Universitario de la Paz (UNIPAZ), Escuela de Ingeniería Agroindustrial, Grupo de Investigación en Innovación, Desarrollo Tecnológico y Competitividad en Sistemas de Producción Agroindustrial (GIADAI). Ingeniera Química. Barrancabermeja, Colombia. https://orcid.org/0000-0003-0804-9450

4 Instituto Universitario de la Paz (UNIPAZ), Escuela de Ingeniería Agroindustrial, Grupo de Investigación en Innovación, Desarrollo Tecnológico y Competitividad en Sistemas de Producción Agroindustrial (GIADAI). Esp. en Ingeniería de Saneamiento Ambiental. Barrancabermeja, Colombia. https://orcid.org/0000-0003-0300-2813

5 Instituto Universitario de la Paz (UNIPAZ), Escuela de Ingeniería Agroindustrial, Grupo de Investigación en Innovación, Desarrollo Tecnológico y Competitividad en Sistemas de Producción Agroindustrial (GIADAI). MSc. en Ingeniería química. Barrancabermeja, Colombia. https://orcid.org/0000-0002-0877-3142 


\section{RESUMEN}

El aceite de Sacha inchi (Plukenetia Volubilis Linneo) es un aceite vegetal que ha despertado gran interés en la producción de biodiesel debido a su baja temperatura de cristalización resultado del alto contenido de PUFAs (ácidos Grasos Poliinsaturados). En la presente investigación se planteó la producción de biodiesel a partir de este aceite, mediante reacciones de transesterificación en una relación aceite: metóxido de potasio de 5:1 v/v. Se realizó una caracterización fisicoquímica del aceite crudo y del biodiesel obtenido, según normas internacionales, y se comparó con la normatividad nacional: NTC 5444. Se realizó paralelamente, una evaluación de la estabilidad oxidativa del biodiesel en contacto con lámina de cobre durante 45 días. Se realizaron mezclas en diferentes proporciones con diésel convencional, con el objeto de evaluar alteración de las propiedades. El rendimiento de reacción para el biodiesel obtenido fue 59,72\%, estos parámetros evaluados se encontraron entre los rangos indicados en la norma. En cuanto a la estabilidad del biodiesel, se encontró evidencia de degradación al contacto con lámina de cobre, trascurridos 28 días. De igual manera, se evidenció que la mejor mezcla a emplear fue B20 (20\% biodiesel de sacha inchi $+80 \%$ diésel), debido a que no presentó alteración significativa de las propiedades del diésel, lo que supone disminución de efectos negativos en motores diésel. Este trabajo proporciona información acerca de la producción de biodiesel de calidad deseable, a partir de aceite de Sacha inchi, con bajos efectos a motores diésel.

\section{ABSTRACT}

Sacha inchi oil (Plukenetia Volubilis Linneo) is a vegetable oil that has awaken great interest in Biodiesel production due to its inherent low crystallization temperature capacity because of high content on PUFAs (Polyunsaturated Fatty Acids). Present research proposed the manufacture of biodiesel using the oil through transesterification reactions, with a $5: 1 \mathrm{v} / \mathrm{v}$ ratio of potassium methoxide. A physicochemical characterization of crude oil, and the biodiesel obtained was carried out according to international standards, as well as NTC 5444 national regulation. Parallel, a 45 days evaluation of the biodiesel oxidative stability when in contact with copper foil was carried out. Different mixtures of conventional diesel were handled in order to evaluate changes in its attributes. Obtained Biodiesel reaction efficiency was 59,72\%, such measurement being within the standard regulations parameters. Regarding the biodiesel stability, evidence of degradation was found in contact with copper foil, after 28 days. Similarly, it was evidenced that the best mixture to use was B20 (20\% sacha inchi biodiesel $+80 \%$ diesel), because it did not show significant alteration on diesel properties, which could mean a reduction of negative

Cómo citar este artículo: BASTO-FLOREZ, LUIS-EDUARDO; MILLÁN-ALVARADO, SEBASTIAN-AUGUSTO; MEDINA-CABALLERO, LUISA-FERNANDA; MORAVERGARA, LIA-ZAMARA; CABALLERO-HERNÁNDEZ, YULEISI-TATIANA. Estudio del biodiesel obtenido a partir de aceite de Sacha inchi (Plukenetia volubilis Linneo). Revista Biotecnología en el Sector Agropecuario y Agroindustrial, v. 20, n. 1, 2022, p. 41-53. Doi: https://doi.org/10.18684/rbsaa.v19.n2.2021.1879

\section{PALABRAS CLAVE:}

Aceite vegetal; Sacha Inchi; Biocombustibles; Biodiesel; Estabilidad oxidativa; FAME; Transesterificación; Ácidos grasos poliinsaturados; Diésel.

\section{KEY WORDS:}

Vegetable oil; Sacha Inchi; Biofuels; Biodiesel; Oxidative stability; FAME; Transesterification; Polyunsaturated fatty acids; Diesel. 
effects in diesel engines. This work provides information about the production of biodiesel of desirable quality, from Sacha inchi oil, with low impact on diesel engines.

\section{INTRODUCCIÓN}

Los sistemas energéticos constituyen componentes amplios y complejos de la economía nacional (Bueno, 2019). En Colombia, la principal fuente de energía es de origen fósil, donde los combustibles obtenidos a partir de estos hidrocarburos se caracterizan por la emisión de gases tóxicos $\left(\mathrm{CO}_{2}, \mathrm{CO}, \mathrm{SO}_{2}\right.$ y NOx) (Andrade et al., 2017). Mitigar estos efectos en la atmósfera es el objetivo de las energías renovables (Dogaeu, 2020), siendo los biocombustibles una alternativa para sustituir total o parcialmente los combustibles convencionales (Lawam, 2018).

El biodiesel en Colombia representa gran potencial en la industria biocarburante (Colmenares et al., 2020). Actualmente, es producido a partir de la transesterificación básica de triglicéridos obtenidos de semillas oleaginosas (Ogunkunle et al., 2019) las cuales pueden ser explotadas en el país puesto que se cuenta con suelos aptos para este tipo de cultivo (Guil et al, 2019). Además, este tipo de combustible no es tóxico, ya que no emite óxidos de azufre ni sustancias nocivas y es biodegradable (Yasar et al., 2020).

En la actualidad, la principal materia prima para la producción de biodiesel es el aceite extraído de palma de aceite y, en Colombia, del híbrido OxG, un cruce entre Elaeis oleifera y Elaeis guineensis (Goh et al., 2017). No obstante, uno de los problemas que la Organización de Laas Naciones Unidas para la Agricultura y la Alimentación (FAO) ha podido detectar es que, ante la alta concentración en la industria de palma de aceite, los cultivos en las plantaciones se están convirtiendo en monovarietales, limitando la resiliencia del cultivo ante las plagas, lo que traduce en un panorama de alto riesgo por la presencia de plagas devastadoras (Sundram et al., 2017).

Por otro lado, aunque el aceite contiene antioxidantes naturales que contribuyen a la estabilidad oxidativa (Zhou et al.,2019), presenta un alto contenido de ácidos grasos libres que en picos de cosecha aumentan, afectando directamente la calidad del combustible (Cong et al., 2020). Además, el terreno en el que se cultivan estas plantas, con el paso de los años se convierte en tierras infértiles, áridas (Malins et al., 2018) presentándose afectación de la biodiversidad (Ocampo et al., 2018).

En la búsqueda de alternativas ante este panorama, se ha despertado interés por el aceite de sacha inchi, un cultivo estratégico en un país tropical con alta humedad, debido a la baja temperatura de cristalización del aceite por su contenido del 82,90 \% en ácidos grasos poliinsaturados (Ramos et al., 2016), comparado con otros cultivos de plantas oleaginosas tropicales (Zuleta et al., 2012; Wang et al., 2018; Kodahl et al., 2020). La presente investigación evaluó el potencial uso del aceite de sacha inchi en mezcla con diésel, bajo estándares de calidad nacionales e internacionales, observándose resultados favorables para algunas concentraciones, dentro de los límites establecidos en la NTC-5444.

\section{MÉTODO}

La presente investigación fue realizada en el Laboratorio de Investigaciones y de Cromatografía del Centro de Investigaciones Santa Lucía, del Instituto Universitario de la Paz, ubicado en la vereda El Zarzal en la ciudad de Barrancabermeja, kilómetro 14 de la vía a Bucaramanga. La temperatura ambiental corresponde a $29-40{ }^{\circ} \mathrm{C}$ y una humedad relativa de $85 \%$. 


\section{Descripción del proceso}

Para la obtención del aceite de Sacha inchi se adecuó la materia prima posterior a la recolección de la semilla en el municipio del Carmen del Chucuri, Santander. Entre las características del cultivo, se tiene que el tiempo de cosecha es de 8 meses a una altura entre los 1.000 y $1.200 \mathrm{~m}$, y requiere un terreno franco arenoso para su cultivo. Posteriormente, se procedió a separar las semillas y realizar limpieza para eliminar impurezas. Seguidamente, se procedió a disminuir el tamaño de estas empleando un molino manual y un secado a $105^{\circ} \mathrm{C}$ durante 24 horas. Finalmente, la extracción del aceite se llevó a cabo por el método soxhlet, empleando hexano como solvente a $60^{\circ} \mathrm{C}$; la mezcla aceite-solvente fue separada empleando destilación al vacío. La caracterización del aceite se realizó mediante pruebas fisicoquímicas.

Una vez caracterizado el aceite, se llevó a cabo la producción de biodiesel mediante reacciones de transesterificación, empleando hidróxido de potasio como catalizador con una relación volumétrica de aceite:metóxido 5:1 durante 180 minutos a $55^{\circ} \mathrm{C}$, en agitación constante (300 rpm); por su parte, para la preparación del metóxido de potasio se emplearon 3,5 g KOH por cada $200 \mathrm{~mL}$ de metanol (Sanchez, 2014).

A continuación, el biodiesel obtenido se dejó en reposo durante 7 días, y se procedió a realizar la separación de la glicerina (fondos) y el sobrenadante (Biodiesel) a temperatura ambiente. El biodiesel obtenido fue caracterizado mediante pruebas fisicoquímicas.

El cálculo de del rendimiento se realizó aplicando la ecuación (1):

$$
\text { Rendimiento }=\frac{\text { volumen de biodiesel }}{\text { volumen de aceite }+ \text { volumen de metóxido }}
$$

Por otro lado, y atendiendo a la problemática de autooxidación que presenta el biodiesel, se evaluó la estabilidad oxidativa del biodiesel; para ello, se tomó como base la norma ASTM-D130, empleando una lámina de cobre con dimensiones aproximadas de $5 \mathrm{~cm}$ de longitud, $1 \mathrm{~cm}$ de ancho y $1 \mathrm{~mm}$ de espesor, adecuadas previamente. Muestras de biodiesel fueron puestas en contacto con las láminas y aisladas de luz y aire, seguidamente se evaluó el comportamiento de las muestras y la solución en periodos de tiempo de 0 a 28 días, por medio de pruebas fisicoquímicas como índice de peróxidos y acidez, asimismo, se evaluó el índice de yodo al inicio y final del tiempo de estudio.

Finalmente, se mezcló el biodiesel obtenido con diésel comercial en concentraciones de 5, 10, 20 y $50 \%$ v/v, con el objeto de evaluar la influencia del biodiesel en las propiedades del diésel.

\section{Parámetros fisicoquímicos}

La calidad y propiedades del aceite empleado y el biodiesel producido se evaluaron mediante pruebas fisicoquímicas basadas en normas AOAC, ASTM y NTC señaladas a continuación:

La evaluación de la viscosidad se realizó mediante el método de prueba estándar para la viscosidad cinemática de líquidos transparentes y opacos (ASTM-D445). La gravedad API se determinó empleando un densímetro automático DMA 4500M (ASTM-D4052). El índice de cetano se halló según la norma ASTM-D4737-10, el índice de cetano se calculó por medio de una ecuación de 4 variables proporcionando un medio para estimar el número de cetano.

El contenido de agua se basó en la norma ASTM-D4377 y ASTM-D1533, se midió en un equipo 870KF Titrino plus y 899 Coulometer, respectivamente. La Temperatura del Punto de inflamación es una medida de la tendencia de un producto para formar una mezcla inflamable con aire bajo las condiciones controladas, empleando el equipo pensky martens copa cerrada según la ASTM-D93 método A. Finalmente, la oxidación se evaluó con base en la norma ASTM-D130. 
El índice de yodo se determinó de acuerdo con la norma A.O.A.C.920.159, empleando reactivo de Wijs, en tanto que el índice de peróxidos según la metodología de la AOAC-965.33, valorando la solución de tiosulfato de sodio 0,01 N.

Para la espectroscopia UV-VIS se empleó el espectrofotómetro Genesys-10S con celdas de cuarzo, como muestra madre se empleó el biodiesel puro para determinar la longitud de onda de mayor absorbancia. Por su parte, el índice de acidez se evaluó siguiendo el procedimiento de la NTC-218, con etanol en caliente y la densidad fue evaluada empleando un picnómetro de Gay-Lussac según la NTC-336.

\section{RESULTADOS}

\section{Caracterización del aceite}

La extracción de aceite de la semilla de sacha Inchi en base seca, partiendo de 5,00 $\pm 0,02$ g de muestra, presentó un rendimiento de $83,62 \%$.

En el Cuadro 1 se presenta la caracterización fisicoquímica del aceite obtenido a partir de la semilla de Sacha inchi que arrojó un contenido de humedad de 0,34\%, significativamente elevado si se tiene en cuenta que este parámetro no debe superar 0,05 \% según la norma ASTM-D675, razón por la cual fue necesario someterlo a un proceso de filtrado con Sulfato de Sodio Anhidro para eliminar dicho exceso, el cual puede influir en la formación de jabones y gomas durante la producción de biodiesel. El contenido de humedad también es alto en comparación con aceite de Jatropha (0,070 \%) (Yate et al., 2020) y aceite de palma (1,50 \%) (Dos Santos et al., 2019). El valor del índice de yodo indica las insaturaciones presentes en los ácidos grasos que conforman los triglicéridos del aceite, un valor de $192,33 \mathrm{~g} \mathrm{I}_{2} / 100 \mathrm{~g}$, es elevado en comparación con el aceite de palma, soja y oliva, los cuales se encuentran alrededor de 55,140 y $95 \mathrm{~g} \mathrm{I}_{2} / 100 \mathrm{~g}$ respectivamente, pero similar a lo reportado por Chasquibol et al. (2016) con 191,9 g I2/100 g y por Follegatti et al. (2009) con $198 \mathrm{~g} \mathrm{I2/100} \mathrm{g,} \mathrm{confirmando} \mathrm{que} \mathrm{el} \mathrm{aceite} \mathrm{de}$ Sacha inchi es de los más insaturados con $93 \%$ del total (Ramos et al., 2019). Un incremento en el índice de yodo conlleva a la disminución del punto de fusión, otorgándole al aceite mayor estabilidad en estado líquido, característica de interés para la producción de biodiesel.

Cuadro 1. Comparación de las propiedades fisicoquímicas del aceite de sacha Inchi.

\begin{tabular}{|c|c|c|c|c|c|}
\hline Propiedad & Unidades & $\begin{array}{l}\text { Aceite crudo } \\
\text { de Sasha Inchi }\end{array}$ & $\begin{array}{l}\text { Aceite crudo de } \\
\text { marañón** }\end{array}$ & $\begin{array}{l}\text { Aceite crudo de } \\
\text { Jatropha curcas*** }\end{array}$ & Norma \\
\hline Densidad a $20^{\circ} \mathrm{C}$ & $\mathrm{g} / \mathrm{cm}^{3}$ & 0,9278 & $0,954^{*}$ & 0,885 & \multirow{4}{*}{ ASTM-D4052 } \\
\hline Densidad a $15^{\circ} \mathrm{C}$ & $\mathrm{kg} / \mathrm{m}^{3}$ & 931,30 & - & - & \\
\hline Densidad a $60^{\circ} \mathrm{F}$ & $\mathrm{kg} / \mathrm{m}^{3}$ & 930,90 & - & - & \\
\hline Gravedad API a $60^{\circ} \mathrm{F}$ & ${ }^{\circ} \mathrm{API}$ & 20,40 & - & - & \\
\hline Índice de yodo & $\mathrm{g} / 2 / 100 \mathrm{~g}$ & 192,33 & 215 & 218 & NTC-283 \\
\hline Índice de refracción & - & 1,478 & 1,693 & 1,468 & NTC-289 \\
\hline Número Ácido & $\mathrm{mg} \mathrm{KOH} / \mathrm{g}$ & 4,335 & 12,1 & 1,63 & ASTM-D664 \\
\hline Índice de Saponificación & $\mathrm{mg} \mathrm{KOH} / \mathrm{g}$ & 187,92 & 47 & 165 & NTC-335 \\
\hline Punto de Fusión & ${ }^{\circ} \mathrm{C}$ & 5,00 & - & - & NTC-213 \\
\hline Contenido de Agua KF & $\%$ Volumen & 0,34 & 3,9 & 0,07 & ASTM-D1533 \\
\hline $\begin{array}{l}\text { Contenido de Agua KF } \\
\text { (Filtrado con Sulfato de } \\
\text { Sodio Anhidro) }\end{array}$ & \% Volumen & 0,12 & - & - & ASTM-D1533 \\
\hline
\end{tabular}


Por otro lado, el índice de saponificación (187,92 mg KOH/g) se encuentra en un valor similar a los aceites de palma, oliva y soja con 199,1, 196 y 191,0 mg KOH/g, respectivamente (Paucaro et al., 2015), este índice está relacionado con la formación de jabones y el índice de acidez. Se observa que este valor se encuentra en los rangos especificados para los aceites empleados en la producción de biodiesel actualmente.

El índice de refracción fue de 1,478 valor similar al reportado por Aranda-Ventura (2019) con 1,48. El número ácido (4,335 mg KOH/g) representa la cantidad de ácidos grasos libres en el aceite, este valor es elevado, luego se puede esperar rancidez con el tiempo (Kiattiphumi et al., 2017), mientras que la densidad de 0,92 coincide con lo reportado por Paucaro y Castaño (2015).

\section{Producción de biodiesel}

Una vez caracterizado el aceite empleado como materia prima, se procedió a la producción de biodiesel; mediante norma EN14103 se determinaron los metilésteres de ácidos grasos (FAME) y la conversión en esteres metílicos, obteniéndose un rendimiento del 59,72 \%, quedando en el biodiesel una concentración elevada $(40,28 \%)$ de triglicéridos propios del aceite que no reaccionaron como se muestra en la figura 1 y posibles trazas de metanol residual, glicerina libre y total que no fueron cuantificadas.

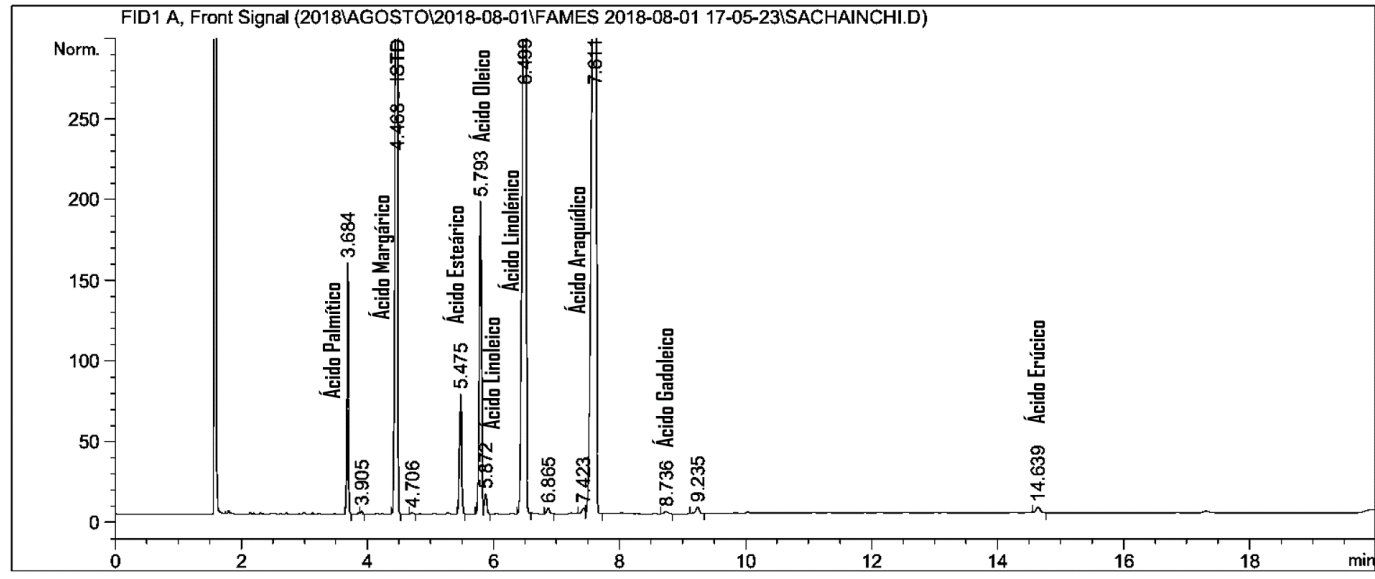

Figura 1. Cromatograma de biodiesel de sacha Inchi:

De acuerdo con los resultados obtenidos (Cuadro 2) se observa que, según la gravedad API (23 ${ }^{\circ} \mathrm{API}$ ), el biodiesel obtenido se clasifica como crudo medio, el cual tienen un rango de densidad de $0,87 \mathrm{a} 0,92 \mathrm{~g} / \mathrm{cm}^{3}$, lo cual concuerda con la densidad obtenida, Por su parte, el punto de inflamación, que es la medida de temperatura a la cual el combustible comenzará a quemarse en contacto con una fuente de calor y, que por tanto, es un parámetro importante para la seguridad, transporte y almacenamiento, se encuentra en $63,5^{\circ} \mathrm{C}$ superior al diésel fósil $\left(52^{\circ} \mathrm{C}\right.$ ) según el Ministerio De Minas Y Energía Y Ministerio De Ambiente Y Desarrollo Sostenible (Colombia, 2021), lo que significa que el biodiesel de Sasha inchi se podría almacenar en mezcla con el diésel, no obstante, el biodiesel de palma registra temperatura de inflamación superior a $120^{\circ} \mathrm{C}$ (Colombia, 2021).

Por otro lado, una combustión de calidad ocurre con una ignición rápida seguida de un quemado total y uniforme del carburante, para ello se requiere un número de cetano de 43 para el diésel, sin embargo, el valor obtenido fue es de 33, por lo cual, al mezclar el biodiesel obtenido, afectaría este parámetro (Arias et al., 2011). El número de cetano pudo disminuir debido a la pérdida de ácidos grasos saturados y una alta proporción de ácidos grasos insaturados; igualmente, la literatura señala que el biodiesel de sacha inchi se caracteriza por poseer números de cetano bajos debido a un contenido de metilésteres poliinsaturados mayores al $60 \%$ que lo hace propenso 
a las reacciones de oxidación (Benjumea et al., 2009; Ramos et al., 2019). El color se encuentra por debajo del límite establecido para diésel de 2 (Colombia, 2021), y es un indicativo del grado de refinación, además, un color claro puede relacionarse con menor posibilidad de oxidación.

Cuadro 2. Comparación de las propiedades fisicoquímicas del biodiesel producido a partir del aceite de Sacha inchi.

\begin{tabular}{|c|c|c|c|c|c|c|}
\hline Propiedad & Unidades & $\begin{array}{l}\text { Biodiesel de } \\
\text { Sacha Inchi }\end{array}$ & $\begin{array}{c}\text { Biodiesel de } \\
\text { marañón * }\end{array}$ & $\begin{array}{c}\text { Biodiesel de } \\
\text { canola* }\end{array}$ & NTC 5444 & Norma \\
\hline Gravedad API & ${ }^{\circ} \mathrm{API}$ & 23,0 & - & - & & \multirow{5}{*}{ ASTM-D4737-10 } \\
\hline $10 \%$ vol recobrado & ${ }^{\circ} \mathrm{C}$ & 253,7 & - & - & & \\
\hline $50 \%$ vol recobrado & ${ }^{\circ} \mathrm{C}$ & 283,4 & - & - & & \\
\hline $90 \%$ vol recobrado & ${ }^{\circ} \mathrm{C}$ & 336,6 & - & - & & \\
\hline Índice de Cetano & & 33,0 & 40 & 53 & 51 mínimo & \\
\hline Punto de Inflamación & ${ }^{\circ} \mathrm{C}$ & 63,5 & 183 & 160 & 120 mínimo & ASTM-D93 \\
\hline Viscosidad & $\mathrm{mm}^{2} / \mathrm{s}$ & 7,874 & 10,66 & 7,22 & $1,9-6$ & ASTM-D445 a $40^{\circ} \mathrm{C}$ \\
\hline Color & & 1,0 & - & - & & ASTM-D1500 \\
\hline Densidad $20^{\circ} \mathrm{C}$ & $\mathrm{g} / \mathrm{cm}^{3}$ & $0,91025^{\circ} \mathrm{C}$ & 0,9089 & 0,9056 & $0,86-0,90$ & ASTM-D4052 \\
\hline Índice de yodo & $\mathrm{gl} / 2 / 100 \mathrm{~g}$ & 54,22 & & & 120 mínimo & NTC-283 \\
\hline Índice de acidez & $\mathrm{mg} \mathrm{KOH} / 100 \mathrm{~g}$ & 0,08 & 0,8 & 0,71 & & ASTM-D664 \\
\hline Contenido de éster & $\%(\mathrm{~m} / \mathrm{m})$ & 59,72 & 55 & 85 & 96,5 & EN14103 \\
\hline
\end{tabular}

Finalmente, la viscosidad es de gran interés para los motores que emplean mezcla con biodiesel ya que suele ser elevado respecto al diésel comercial (1,9-5,0 mm²/s), como es el caso del B100 que, según la Resolución 40103 del 2021 se encuentra en un rango entre 3,5 y $5 \mathrm{~mm}^{2} / \mathrm{s}$ ) (Colombia, 2021), mientras que la viscosidad del biodiesel objeto de estudio $\left(7,874 \mathrm{~mm}^{2} / \mathrm{s}\right)$ es elevada, lo que podría generar problemas en los sistemas de inyección e insuficiencia en el proceso de atomización y puede deberse al incremento de saturaciones y longitud de la cadena carbonada de los ácidos grasos libres (Niraj, 2017), evidenciando que no tendría un uso comercial puro. Para reducir la viscosidad se puede realizar mezclado del biodiesel en bajas proporciones con diésel convencional, mezclas de aceites que contienen diferentes composiciones de ácidos grasos o usar aditivos formulados para mejorar las propiedades de flujo a baja temperatura (Benavides et al., 2021).

\section{Evaluación de la estabilidad oxidativa}

La estabilidad oxidativa del biodiesel se determinó mediante pruebas fisicoquímicas, relacionando el índice de acidez, índice de peróxidos e índice de yodo durante 28 días (Figura 2a y 2b).
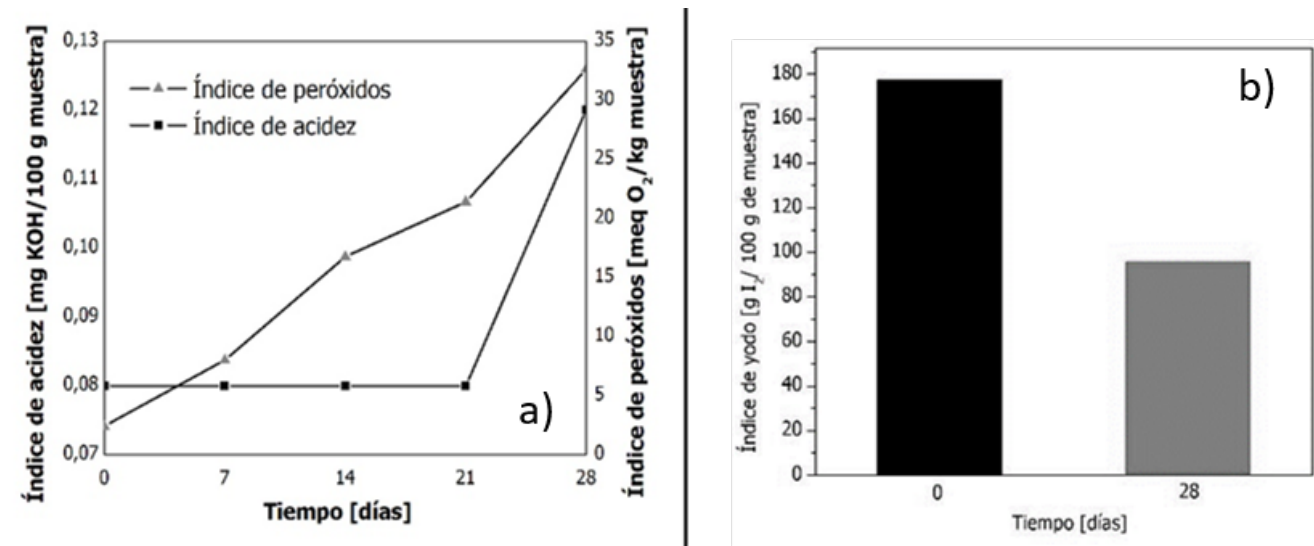

Figura 2. a) Índice de acidez e Îndice de peróxidos. b) Índice de yodo. 
Se observa un incremento constante del índice de peróxidos en el tiempo de estudio, asociado a la degradación que sufren los ácidos grasos insaturados en las fases iniciación, propagación y terminación, que dan lugar a hidroperóxidos (Chee et al., 2020). Por su parte, la estabilidad del índice de acidez durante los primeros 21 días se puede asociar al tiempo de formación de complejos metálicos que impiden la interacción entre el metal y los hidroxiperóxidos formados durante el proceso de degradación de los ésteres metílicos, sin embargo, se puede presentar concentración de nuevos productos en la solución, que conllevan al aumento en la acidez como se evidencia a partir del día 21.

La disminución del índice de yodo se puede relacionar con la auto oxidación de los ésteres metílicos que se producen como consecuencia de las reacciones en cadena de radicales libres debido a los ácidos grasos insaturados durante las fases de iniciación, propagación y terminación. El radical lipídico se forma a partir del ataque de radicales, luz, calor o por las trazas de cobre, seguidamente reacciona con oxígeno generando un radical peroxilo (ROO-), que ataca la matriz base, dando lugar a la formación de hidroperóxidos lipídicos ( $\mathrm{ROOH}$ y un nuevo radical que inicia de nuevo la secuencia de propagación. Teniendo en cuenta que el índice de yodo evalúa la presencia de insaturaciones y éstas disminuyen en el proceso de degradación, su disminución concuerda con la teoría de oxidación y con el comportamiento del índice de acidez y peróxidos (Reddy et al., 2018; Sia et al., 2020). Con objeto de evaluar la degradación de los esteres metílicos de Sacha inchi se realizaron mediciones de absorbancia a través del tiempo, a 455 nm (longitud de onda de mayor absorbancia).

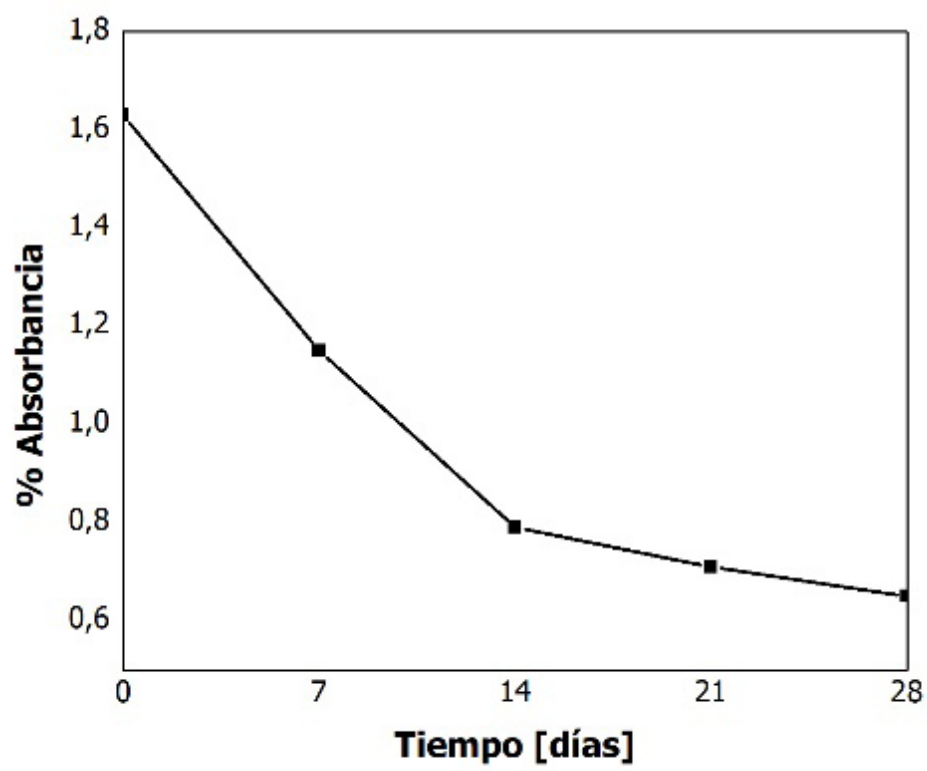

Figura 3. Porcentaje de Absorbancia.

En la Figura 3 se observa que la absorbancia disminuye ligeramente, lo cual se puede asociar a la tonalidad opaca que tomó la muestra a medida que aumentaba el tiempo de estudio, así como a la degradación de los ésteres metílicos en contacto con lámina de cobre.

Según lo establecido en la norma ASTM-D130 para determinar el índice de corrosión, en la Figura 4 se presentan las láminas de cobre que estuvieron sumergidas en el biodiesel, observándose que no hubo una variación significativa en ellas, encontrándose en la franja 1B de naranja oscuro (según la clasificación del cuadro 1 de ASTM-D13), evidenciando que no hay ocurrencia de un proceso de corrosión representativo en el tiempo de estudio, pero si la formación de mancha ligeras que se pueden asociar a complejos metálicos. 


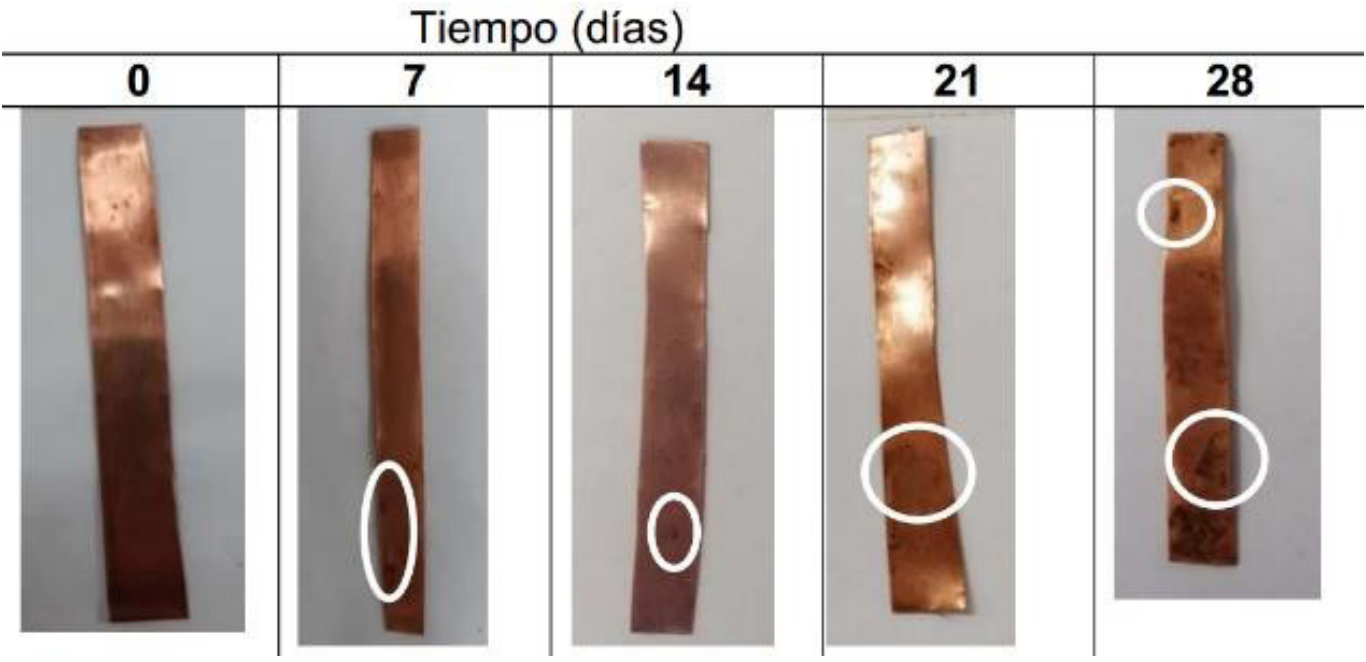

Figura 4. Corrosión en las láminas de cobre.

\section{Mezcla diésel - biodiesel}

Finalmente, se procedió a evaluar el comportamiento del diésel en mezcla con el biodiesel de aceite de Sacha inchi en diferentes porcentajes de mezcla, B5, B10, B20 y B50.

En la Figura 5, se observa el comportamiento de la densidad, gravedad API y viscosidad de la mezcla diésel-biodiesel en diferentes proporciones, observándose que, debido a que la densidad del biodiesel es mayor a la del diésel, esta aumenta gradualmente en relación con el incremento de la concentración en la mezcla. De igual manera, la viscosidad presenta un incremento progresivo esperado, debido a que la viscosidad del biodiesel es superior a la del diésel. Por su parte, las muestras presentan entre 32 y $29^{\circ} \mathrm{API}$, catalogándose como crudo mediano (Espitia et al., 2019) y se resalta que los valores para estos parámetros en la mezcla se mantienen en el rango estipulado en la norma NTC 5444.

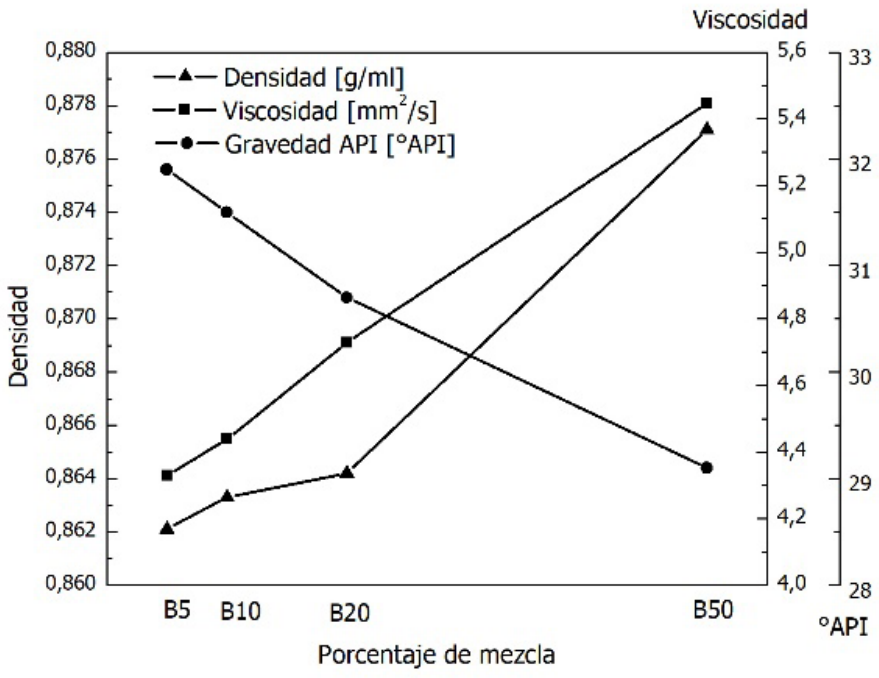

Figura 5. Densidad, Gravedad API y Viscosidad de la mezcla diésel-biodiesel 
En la Figura 6a se observa el comportamiento del índice de acidez e índice de yodo de la mezcla diésel-biodiesel para diferentes concentraciones, encontrándose que la acidez aumentó de manera proporcional a la concentración de biodiesel en mezcla, debido a que la acidez de los ésteres metílicos es superior a la del diésel, como resultado de los ácidos grasos libres producto de la hidrólisis. Sin embargo, este parámetro se mantiene en el rango estipulado para la mezcla comercial, no obstante, debido a que el aumento de acidez puede indicar degradación oxidativa, se recomienda estudiar la influencia del tiempo y temperatura de almacenamiento (Niraj, 2017).

Por otro lado, se observa que los valores del índice de yodo de las muestras superan los parámetros establecidos en la NTC 5444, donde el máximo permitido es de $120 \mathrm{~g} \mathrm{I} / 100 \mathrm{~g}$. Autores como Federico Ganduglia y su equipo de investigación para el manual de biocombustible del IICA del Perú (2009), plantean qu,e a medida que los aceites poseen en su composición mayor proporción de ácidos grasos insaturados, presentan un menor número de cetano y menor estabilidad, lo que se traduce en un mayor Índice de Yodo, lo que define al aceite de Sacha inchi como unos de los aceites más insaturados.
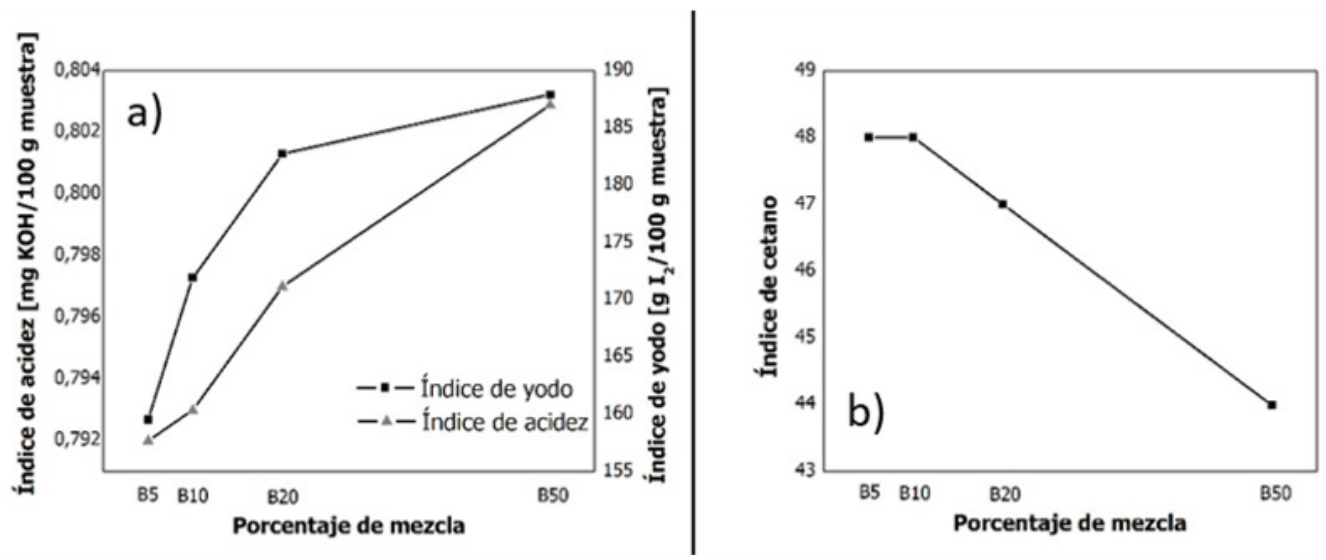

Figura 6. a) Índice de acidez e Índice de yodo y b) Índice de cetano para la mezcla diésel- biodiesel.

Finalmente, la Figura 6b muestra el comportamiento del índice de cetano para la mezcla diésel-biodiésel, observándose que este parámetro disminuye proporcional al incremento porcentual en la muestra. Un número de cetano por debajo de 47 está fuera de los parámetros establecidos para el biodiesel, y es indicativo de la baja calidad de ignición de un combustible diésel, lo que puede provocar el llamado "golpeteo diésel" que hace referencia a la demora en el inicio de la combustión. Los resultados anteriores conllevan a concluir que el biodiesel producido a partir del aceite de Sacha inchi bajo los parámetros de estudio, solo se podría mezclar con diésel hasta un $20 \%$ v/v, sin alterar significativamente los parámetros de calidad del diésel.

\section{CONCLUSIONES}

El aceite obtenido de la semilla de Sacha inchi presenta elevada humedad que puede traer consecuencia en la producción de biodiesel por lo que se hizo necesario someter el aceite a secado para disminuir la posibilidad de formación de jabones en las reacciones de transesterificación y dificultar el proceso de producción de biodiesel.

El biodiesel obtenido a partir del aceite de Sacha inchi presentó bajo rendimiento en las reacciones de transesterificación, sin embargo, sus propiedades fisicoquímicas se encuentran entre los parámetros establecidos en la NTC 5444. De igual manera, se observa que en los 28 días de estudio se evidencia degradación del biodiesel en contacto con lámina de cobre, no obstante, se puede evaluar la influencia de antioxidantes que incrementen el tiempo de inducción del combustible y retrasen el proceso de degradación. Igualmente, se podría aumentar la proporción de ácidos grasos monoinsaturados y generar un balance entre la estabilidad oxidativa y las pro- 
piedades de flujo a baja temperatura y optimizar el perfil de ácidos grasos mezclando biodiesel de diferentes materias primas. Finalmente, en mezcla con diésel convencional, se puede concluir que el biodiesel a partir de aceite de Sacha inchi se podría mezclar hasta porcentajes inferiores al $20 \%$, sin modificar de forma significativa los parámetros de calidad del diésel.

\section{AGRADECIMIENTOS}

Los autores agradecen al Instituto Universitario de la Paz -UNIPAZ, a la Escuela de Ingeniería Agroindustrial y al Grupo de Investigación GIADAI, por el apoyo en la presente investigación.

\section{REFERENCIAS}

ANDRADE-CASTAÑEDA, HERNÁN-JAIR; ARTEAGA-CÉSPEDES, CRISTHIAN-CAMILO; SEGURAMADRIGAL, MILENA-ANDREA. Emisión de gases de efecto invernadero por uso de combustibles fósiles en Ibagué, Tolima (Colombia). Corpoica Ciencia y Tecnología Agropecuaria, v. 18, n. 1, 2017, p. 102-112. https://doi.org/10.21930/rcta.vol18_num1_art:561

ARANDA-VENTURA, JOSÉ; VILLACRÉS-VALLEJO, JORGE; RIOS-ISERN, FELIPE. Composición química, características físico-químicas, trazas metálicas y evaluación genotóxica del aceite de Plukenetia volubilis $L$. (sacha inchi). Revista peruana de medicina integrativa, v. 4, n. 1, 2019, p. 4-14. http://dx.doi.org/10.26722/rpmi.2019.41.103

ARIAS-PÉREZ, DANIEL-EDUARDO; TEUTA-RAMÍREZ, CÉSAR-AUGUSTO; PARRA-PLAZAS, JAIME. Caracterización de las propiedades del biodiesel de girasol bajo la Norma NTC de 100/04 y medición de poder calorífico. Avances Investigación en Ingeniería, v. 8, n. 2, 2011, p. 73-80. https://revistas.unilibre.edu.co/index.php/avances/article/view/2709

BENAVIDES, ALIRIO-YOVANY; BENJUMEA, PEDRO-NE; AGUDELO, JOHN-RAMIRO. EI fraccionamiento por cristalización del biodiesel de aceite de palma como alternativa para mejorar sus propiedades de flujo a baja temperatura. Revista Facultad de Ingeniería Universidad de Antioquia, v. 43, 2008, p. 7-17. http://www.scielo.org.co/scielo.php?script=sci_arttext\&pid=S0120-62302008000100001\&lng=en\&nrm=iso

BENJUMEA-HERNÁNDEZ, PEDRO-NEL; AGUDELO-SANTAMARÍA, JOHN-RAMIRO; RIOS, LUIS-ALBERTO. Biodiésel: Producción, calidad y caracterización. Medellín (Colombia): Universidad de Antioquia, 2009, 152 p.

BUENO-LÓPEZ, MAXIMILIANO. La evolución de los sistemas energéticos: retos y oportunidades para el sistema eléctrico colombiano. Ámbito Investigativo, v. 4, n. 3, 2019, p. 22-31.

CASTAÑO, DIEGO-LEANDRO; VALENCIA, MARIA-DEL-PILAR; MURILLO, ELIZABETH; MENDEZ, JONHJAIRO; ERAS-JOLI, JORDI. composición de ácidos grasos de sacha inchi (Plukenetia volúbilis Linneo) y su relación con la bioactividad del vegetal. Revista chilena de nutrición, v. 39, n. 1, 2012, p. 45-52. http://dx.doi.org/10.4067/S0717-75182012000100005

CHASQUIBOL, N.A.; GÓMEZ-COCA, R.B.; YÁCONO, J.C.; GUINDA, Á.; MOREDA, W.; AGUILA, C.; CAMINO, M.C. Markers of quality and genuineness of commercial extra virgin sacha inchi oils. Grasas y Aceites, v. 67, n. 4, 2016, e169. https://doi.org/10.3989/gya.0457161

COLMENARES-QUINTERO, RAMÓN-FERNANDO; RICO-CRUZ, CAMILO-JOSÉ; STANSFIELD, KIM E.; COLMENARES-QUINTERO, JUAN-CARLOS. Assessment of biofuels production in Colombia. Cogent Engineering, v. 7, n. 1, 2020, e1740041. https://doi.org/10.1080/23311916.2020.1740041 
COLOMBIA. MINISTERIO DE MINASYENERGÍA YMINISTERIODEAMBIENTEYDESARROLLOSOSTENIBLE. Resolución 40103. Por la cual se establece los parámetros y requisitos de la calidad del combustible diésel (ACPM), los biocombustibles para uso en motores encendidos por compresión como componentes de mezcla en procesos de combustión y de sus mezclas y, de las gasolinas básicas y gasolinas oxigenadas con etanol anhidro combustible para uso en motores de encendido por chispa, y se adoptan otras disposiciones. Bogotá (Colombia): 2021, 15 p.

DOGARU, LUCRETIA. The Main Goals of the Fourth Industrial Revolution. Renewable Energy Perspectives. Procedia manufacturing, v. 46, 2020, p. 397-401 https://doi.org/10.1016/j.promfg.2020.03.058

DOS SANTOS, LETÍCIA-KAREN; RODRIGUES-HATANAKA, RAFAEL; DE OLIVEIRA, JOSÉ-EDUARDO; FLUMIGNAN, DANILO-LUIZ. Production of biodiesel from crude palm oil by a sequential hydrolysis/ esterification process using subcritical water. Renewable Energy, v. 130, 2019, p. 633-640. https://doi.org/10.1016/j.renene.2018.06.102

ESPITIA-CUBILLOS, ANNY-ASTRID; DELGADO-TOBÓN, ARNOLDO-EMILIO; CAMARGO-VARGAS, SERGIOALEJANDRO. Estimación teórica del efecto de la temperatura en la densidad, viscosidad, poder calorífico, capacidad calorífica y gravedad API de biocombustibles. Scientia et Technica, v. 24, n. 2, 2019, p. 190-199.

FOLLEGATTI-ROMERO, LUIS A.; PIANTINO, CARLA R.; GRIMALDI, RENATO; CABRAL, FERNANDO A. Supercritical $\mathrm{CO}_{2}$ extraction of omega-3 rich oil from Sacha inchi (Plukenetia volubilis L.) seeds. The Journal of Supercritical Fluids, v. 49, n. 3, 2009, p. 323-329. https://doi.org/10.1016/j.supflu.2009.03.010

GANDUGLIA, FEDERICO. Manual de Biocombustibles. Buenos Aires (Argentina): IICA-ARPEL, 2009, 230 p.

GE, JUN-CONG; KIM, HO-YOUNG; YOON, SAM-KI; CHOI, NAG-JUNG. Optimization of palm oil biodiesel blends and engine operating parameters to improve performance and PM morphology in a common rail direct injection diesel engine. Fuel, v. 260, 2020, e116326.

https://doi.org/10.1016/j.fuel.2019.116326GOH, K.J.; WONG, C.K.; NG, P.H.C. Oil Palm. Encyclopedia of Applied Plant Sciences, https://doi.org/10.1016/B978-0-12-394807-6.00176-3

GUIL-LAYNEZ, JOSÉ-LUIS; GUIL-GUERRERO, JOSÉ-LUIS; GUIL-LAYNEZ, ALVARO. Bioprospecting for seed oils in tropical areas for biodiesel production. Industrial Crops and Products, v. 128, 2019, p. 504-511. https://doi.org/10.1016/j.indcrop.2018.11.044

KODAHL, NETE. Sacha inchi (Plukenetia volubilis L.) from lost crop of the Incas to part of the solution to global challenges?. Planta, v. 251, n. 4, 2020, p. 22. https://doi.org/10.1007/s00425-020-03377-3

KUMAR, NIRAJ. Oxidative stability of biodiesel: Causes, effects and prevention. Fuel, v. 190, 2017, p. 328-350. https://doi.org/10.1016/j.fuel.2016.11.001

LAWAM-MUHAMMAD, USMAN; MUHAMMAD-SHAMSUDDIN, IBRAHIM; DANJUMA, ABDURRAHMAN; SULAIMAN-MUSAWA, RABI'U; HAFSAT-DEMBO, UMAR. Biofuels as the Starring Substitute to Fossil Fuels. Petroleum Science and Engineering, v. 2, n. 1, 2018, p. 44-49. https://doi.org/10.11648/j.pse.20180201.17

MALINS, C. Driving deforestation: The impact of expanding palm oil demand through biofuel policy. Rainforest Foundation Norway Cerulogy. 2018. https://www.cerulogy.com/wp-content/uploads/2018/02/Cerulogy Driving-deforestation_Jan2018.pdf [consultado marzo 23 de 2021]

OCAMPO-PEÑUELA, NATALIA; GARCÍA-ULLOA, JOHN; GHAZOUL, JABOURY; ETTER, ANDRES. Quantifying impacts of oil palm expansion on Colombia's threatened biodiversity. Biological Conservation, v. 224 2018, p. 117-12. https://doi.org/10.1016/j.biocon.2018.05.024

OGUNKUNLE, OYETOLA; AHMED, NOOR A. review of global current scenario of biodiesel adoption and combustion in vehicular diesel engines. Energy Reports, v. 5, 2019, p. 1560-1579.

https://doi.org/10.1016/j.egyr.2019.10.028 
PAUCARO-MENACHO, LUZ; SALVADOR-REYES, REBECA; GUILLÉN-SANCHEZ, JHOSELINE; CAPA-ROBLES, JUAN; MORENO-ROJO, CESAR. Estudio comparativo de las características físico-químicas del aceite de sacha inchi (Plukenetia volubilis I.), aceite de oliva (Olea europaea) y aceite crudo de pescado. Scientia Agropecuaria, v. 6, n. 4, 2015, p. 279-290.

https://doi.org/10.17268/sci.agropecu.2015.04.05

RAMOS-ESCUDERO, FERNANDO; MUÑOZ, ANA-MARÍA; RAMOS-ESCUDERO, MONICA; VIÑAS-OSPINA, ADRIANA; MORALES, MARIA-TERESA; ASUERO, AGUSTÍN G. Characterization of commercial Sacha inchi oil according to its composition: tocopherols, fatty acids, sterols, triterpene and aliphatic alcohols. Joournal Of Food Science and Technology, v. 56, 2019, p. 4503-4515. https://doi.org/10.1007/s13197-019-03938-9

RAMOS-ESCUDERO, FERNANDO; PONCE-DAYER, LADY; BARNETT-MENDOZA, EDY; CELI-SAAVEDRA, LUIS; RAMOS-ESCUDERO, MÓNICA. Perfil de ácidos grasos de aceite de Sacha inchi (Plukenetia volubilis) en comparación con otros aceites vírgenes comestibles. Revista Campus, v. 21, n. 21, 2016, p. 101-108.

REDDY, A.N.R.; SALEH, A.A.; ISLAM, M.S.; RAHMAN, M.R.; MASJUKI, H.H. Experimental evaluation of fatty acid composition influence on Jatropha biodiesel physicochemical properties. Journal of renewable and sustainable energy, v. 10, n. 1, 2018, e013103. https://doi.org/10.1063/1.5018743

SAENGSORN, KIATTIPHUMI; JIMTAISONG, AMPA. Determination of hydrophilic-lipophilic balance value and emulsion properties of sacha inchi oil. Asian Pacific Journal of Tropical Biomedicine, v. 7, n. 12, 2017, p. 1092-1096. https://doi.org/10.1016/j.apjtb.2017.10.011

SANCHEZ-TORRES, JOSÉ-ALCIDES. Determinación de la relación entre parámetros de proceso y rendimiento de obtención de biodiesel a partir de aceites de cocina usados, con base en meta-análisis [Tesis de Maestría en Diseño y Gestión de Procesos]. Bogotá (Colombia): Universidad de la Sabana, Facultad de ingeniería, 2014, $171 \mathrm{p}$.

SIA, CHEE-BING; KANSEDO, JIBRAIL; TAN, YIE-HUA; LEE, KEAT-TEONG. Evaluation on biodiesel cold flow properties, oxidative stability and enhancement strategies: A review. Biocatalysis and Agricultural Biotechnology, v 24, 2020, e101514. https://doi.org/10.1016/j.bcab.2020.101514

SUNDRAM, SHAMALA; INTAN-NUR, A.M. South American Bud rot: A biosecurity threat to South East Asian oil palm. Crop Protection, v. 101, 2017, p. 58-67. https://doi.org/10.1016/j.cropro.2017.07.010

WANG, SUNAN; ZHU, FAN; KAKUDA, YUKIO. Sacha inchi (Plukenetia volubilis L.): Nutritional composition, biological activity, and uses. Food Chemistry, v. 265, 2018, p. 316-328. https://doi.org/10.1016/j. foodchem.2018.05.055

YASAR, FEVZI. Comparison of fuel properties of biodiesel fuels produced from different oils to determine the most suitable feedstock type. Fuel, v. 264, 2020, e116817. https://doi.org/10.1016/j.fuel.2019.116817

YATE, ANDREA V.; NARVÁEZ, PAULO C.; ORJUELA, ALVARO; HERNÁNDEZ, ADRIANA; ACEVEDO, HELMER. A systematic evaluation of the mechanical extraction of Jatropha curcas $L$. oil for biofuels production. Food and Bioproducts Processing, v. 122, 2020, p. 72-81.

https://doi.org/10.1016/j.fbp.2020.04.001

ZHOU, JIEXIN; MA, YANLI; JIA, YIJIA; PANG, MINGJIE; CHENG, GUIGUANG; CAI, SHENGBAO. Phenolic profiles, antioxidant activities and cytoprotective effects of different phenolic fractions from oil palm (Elaeis guineensis Jacq.) fruits treated by ultra-high pressure. Food Chemistry, v. 288, 2019, p. 68-77. https://doi.org/10.1016/j.foodchem.2019.03.002

ZULETA, ERNESTO C.; RIOS, LUIS A.; BENJUMEA, PEDRO-N. Oxidative stability and cold flow behavior of palm, sacha-inchi, jatropha and castor oil biodiesel blends. Fuel Processing Technology, v. 102, 2012, p. 96-101.

https://doi.org/10.1016/j.fuproc.2012.04.018 